\title{
Dinâmica populacional do psilídeo-de-concha Glycaspis brimblecombei (Moore, 1964) (Hemiptera: Psyllidae) e de seu parasitóide Psyllaephagus bliteus (Hymenoptera: Encyrtidae) em floresta de Eucalyptus camaldulensis
}

\author{
Population dinamics of red gum lerp psyllid, Glycaspis brimblecombei (Moore, 1964) \\ (Hemiptera: Psyllidae) and its parasitoid, Psyllaephagus bliteus (Hymenoptera: Encyrtidae), \\ in Eucalyptus camaldulensis plantation

\begin{abstract}
Pedro José Ferreira Filho ${ }^{\mathrm{I}^{*}}$ Carlos Frederico Wilcken ${ }^{\mathrm{I}}$ Nádia Cristina de Oliveira ${ }^{\mathrm{I}}$ Mário Henrique Ferreira do Amaral Dal Pogetto ${ }^{I}$ Alexandre Coutinho Vianna Lima ${ }^{I}$
\end{abstract}

RESUMO

O psilídeo-de-concha, Glycaspis brimblecombei, é uma praga exótica específica de Eucalyptus, que causa desfolha, secamento de ponteiros e ramos, podendo levar as árvores à morte. O parasitóide Psyllaephagus bliteus é indicado como principal agente no controle biológico desta praga. Portanto, o monitoramento adequado de as ambas espécies por um método eficiente de amostragem facilitaria o manejo para uma futura tomada de decisão. Devido à falta de informações referentes ao seu comportamento em plantações extensivas de eucalipto no Brasil, este trabalho teve como objetivo estudar as populações de G. brimblecombei e de P. bliteus em florestas de Eucalyptus camaldulensis, utilizando armadilhas amarelas para determinação de sua flutuação populacional e correlacionado-as com variáveis meteorológicas (temperatura e precipitação). A determinação da flutuação populacional (53 pontos amostrais) foi realizada em talhão de 19ha com E. camaldulensis em Luíz Antônio, SP, em 10avaliações quinzenais, entre janeiro e junho de 2005. As populações de ambas as espécies apresentaram correlação inversamente proporcional em função da temperatura e não há correlação com a precipitação no período avaliado.

Palavras-chave: eucalipto, praga florestal, inimigo natural, controle biológico, proteção florestal.

\section{ABSTRACT}

Psyllids are one most widespread group of pests, causing severe damage on agricultural and forestry systems. The recently discover about occurrence of red gum lerp psyllid Glycaspis brimblecombei Moore (Hemiptera: Psyllidae), in Brazil is, nowadays a serious problem in Eucalyptus forests, mainly in $\boldsymbol{E}$. camaldulensis. The psyllid specific parasitic wasp Psyllaephagus bliteus Riek (Hymenoptera: Encyrtidae) is the main biological control agent of this pest. Thus, the adequate monitoring of both species by efficient sampling method would facilitate a future decision making. Due to economic importance, this study aimed to evaluate G. brimblecombei and P. bliteus populations in Eucalyptus camaldulensis plantation, using yellow traps to determinate population fluctuation with weather interactions (temperature and rainfall). The study of the pest and parasitoid population (53 sampling dates) was carried out on E. camaldulensis plantations in 19 ha area in Luíz Antônio municipality (SP, Brazil), with quarterly samplings, between January and June, 2005 (ten sampling dates). This research indicated that populations had an inversely proportional correlation subject according to the temperature. However, there is no correlation between both species with the rainfall in the evaluated period.

Key words: eucalypt, forest pest, natural enemy, biological control, forest protection.

\section{INTRODUÇÃO}

O psilídeo-de-concha, Glycaspis brimblecombei, é uma espécie pertencente à ordem Hemiptera, subordem Sternorrhyncha e família Psyllidae. Os psilídeos são insetos pequenos, semelhantes a pequenas cigarrinhas e de hábito sugador (GALLO et al., 2002). Entre os psilídeos associados ao eucalipto, os gêneros mais importantes são Cteranytaina, Blastopsylla, Creiis, Eucalyptolyma, Cardiaspina e Glycaspis (ELLIOTT et al., 1998).

O monitoramento de $\boldsymbol{G}$. brimblecombei é importante para se conhecer seus níveis de infestação por meio do tempo e identificar os fatores que podem

'Departamento de Produção Vegetal, Setor de Defesa Fitossanitária, Faculdade de Ciências Agronômicas (FCA), Universidade Estadual Paulista (UNESP), Rua José Barbosa de Barros, 1780, CP 237, 18610-307, Botucatu, SP, Brasil. E-mail: pedroferreira@fca.unesp.br. *Autor para correspondência. 
influenciar em sua população. Nos EUA e no México, o monitoramento de psilídeos em plantios de eucalipto utilizados para arborização urbana é realizado com armadilhas adesivas de coloração amarela, confeccionadas a partir de tampas circulares, cobertas com óleo de motor para tornarem-se adesivas (CIBRIÁN-TOVAR et al., sd; DAHLSTEN et al., 1998).

Na Austrália, em florestas de Eucalyptus camaldulensis, o psilídeo-de-concha $G$. brimblecombei apresenta de duas a quatro gerações por ano. Já no México, é seguro afirmar que o número de gerações é maior, especialmente nos meses secos e quentes da primavera e do verão (CIBRIÁN-TOVAR \& IÑIGUEZ-HERRERA, 2001).

Os danos causados pelo psilídeo-de-concha G. brimblecombei podem ser de grande proporção, já que chegam a apresentar $15 \%$ de mortalidade no primeiro ano e até $40 \%$ no segundo ano, se não forem realizados métodos de controle (GILL, 1998). DREISTADT \& GILL (1999) perceberam que seu dano se dava por meio da alimentação tanto das ninfas como dos adultos, extraindo as substâncias que se encontram nas folhas.

Psyllaephagus é um gênero com algumas espécies de importância no controle de psilídeos que atacam mirtáceas. Na Califórnia, EUA, foram introduzidas da Austrália as espécies: Psyllaephagus pilosus, para o controle de Cteranytaina eucalypti em Eucalyptus pulverulenta e Psyllaephagus bliteus para o controle de $\boldsymbol{G}$ brimblecombei em $\boldsymbol{E}$. camaldulensis (BERTI-FILHO et al., 2003).

A espécie $\boldsymbol{P}$. bliteus é uma vespa pertencente à ordem Hymenoptera, subordem Apocrita e família Encyrtidae e se caracteriza por ser parasitóide específico de $\boldsymbol{G}$ brimblecombei. Os insetos pertencentes a essa família são microhimenópteros de 1 a $2 \mathrm{~mm}$, caracterizados pela mesopleura larga e convexa, sendo que a maioria das espécies são parasitóides de pulgões (GALLO et al., 2002).

Em razão dos danos verificados pela introdução de $\boldsymbol{G}$ brimblecombei nos EUA e no México (DAHLSTEN et al., 2003) e da falta de informações referente ao seu comportamento em plantações extensivas de eucalipto no Brasil, foi realizado este trabalho a fim de determinar a flutuação populacional de $\boldsymbol{G}$ brimblecombei e de $\boldsymbol{P}$. bliteus em floresta de $\boldsymbol{E}$. camaldulensis por meio de armadilhas amarelas.

\section{MATERIAL E MÉTODOS}

O trabalho foi desenvolvido na Fazenda Cara Preta, pertencente à Votorantim Celulose e Papel, localizada no município de Luiz Antônio, São Paulo, (SP), com as seguintes coordenadas planas: $228720 \mathrm{~S}$ 7613149 E, com altitude média de 729m. Foi utilizado um talhão com plantio de $\boldsymbol{E}$. camaldulensis, com 6,5 anos de idade, espaçamento de 2,5 x 3,0 metros, com área aproximada de 19 hectares, com ocorrência do psilídeo-de-concha desde 2003.

O levantamento populacional dos adultos de $\boldsymbol{G}$ brimblecombei e de $\boldsymbol{P}$. bliteus foi realizado com armadilhas, que consistiram de cartões plásticos amarelos, com adesivo em ambas as faces, nas medidas de 10 x 12cm, (BIOTRAP ${ }^{\circledR}$ amarela). Foram instaladas 86 armadilhas amarelas distribuídas uniformemente no talhão, em malha de 50 x 36m, e altura aproximada de 1,80 metros do solo entre duas árvores.

Foram realizadas 10 coletas na área e, a intervalos médios de aproximadamente 15 dias, as armadilhas foram recolhidas, identificadas e embaladas com filme plástico transparente para não danificar os insetos capturados e facilitar a identificação. Após a coleta, as armadilhas foram levadas ao laboratório para ser feita a triagem e a contagem dos insetos. Nas armadilhas amarelas, foi feita a contagem do número de machos, de fêmeas e total de adultos de $\boldsymbol{G}$. brimblecombei e de $\boldsymbol{P}$. bliteus, nas duas faces das armadilhas. A flutuação populacional de $\boldsymbol{G}$. brimblecombei e de $\boldsymbol{P}$. bliteus foi determinada graficamente, considerando-se os resultados obtidos nas armadilhas ao longo de um período observado de seis meses.

Com a finalidade de subsidiar o entendimento dos resultados obtidos, foram utilizados os seguintes elementos meteorológicos: temperaturas médias, máximas e mínimas e pluviosidade. As leituras foram diárias, no entanto, para a apresentação dos resultados, foram considerados os valores médios dos intervalos entre cada coleta. Os dados foram obtidos junto à estação meteorológica instalada no viveiro florestal da empresa, na mesma fazenda onde foi instalado o experimento.

Foram realizadas análises exploratórias independentes para cada data de avaliação, considerando-se a média e o teste de correlação linear de Pearson entre o número médio de $\mathbf{G}$ brimblecombei e de $\boldsymbol{P}$. bliteus de cada unidade amostral com os dados meteorológicos.

\section{RESULTADOS E DISCUSSÃO}

Flutuação populacional de $\boldsymbol{G}$ brimblecombei e de $\boldsymbol{P}$. bliteus

O número médio de adultos de $\boldsymbol{G}$. brimblecombei e de $\boldsymbol{P}$. bliteus capturados nas 
armadilhas amarelas apresentaram-se bastante similares em visão generalizada, pois, desde o início do experimento, as duas espécies tiveram aumento do número de indivíduos a cada avaliação.

A variação no número médio de adultos de G. brimblecombei capturados nas 86 armadilhas amarelas desde a instalação até o final do experimento foi de 0,34 a 132,58 psilídeos por armadilha. Para a espécie $\boldsymbol{P}$. bliteus, o número médio de insetos capturados nas armadilhas foram menores, variando de 0,14 a 34,51 parasitóides por armadilha (Figura 1), sendo os valores mínimos e máximos absolutos obtidos por período de avaliação de 29 (15/02/05) e 15750 (01/ 06/05) psilídeos e de 3 (31/03/05) e 3203 (01/06/05) parasitóides respectivamente.

Para o número médio de adultos de $\boldsymbol{G}$. brimblecombei, foi observada, desde o início das coletas, tendência crescente na quantidade de indivíduos capturados por armadilha, atingindo pico após 124 dias de avaliação, quando foram observadas médias de 183,14 psilídeos por armadilha. Isso provavelmente tenha ocorrido devido ao início do período seco, coincidindo com período do início de altas infestações da praga e principalmente devido ao período em que a armadilha ficou exposta a campo (20 dias), sendo que o tempo médio de exposição das armadilhas a cada avaliação foi de 14 a 15 dias. Isso ocorreu devido à data de avaliação ter coincidido com dias de chuva intensa, que impossibilitou a realização da coleta. Portanto, este pico populacional aos 124 dias de avaliação (01/06/05) não foi o pico máximo de infestação, pois, quando as avaliações foram encerradas, o número de insetos capturados por armadilha estava aumentando a cada avaliação.

A mesma tendência crescente na quantidade de parasitóides capturados por armadilha pôde ser observada, porém, com uma queda após 62 dias de avaliação (31/03/05), atingindo um pico juntamente com o número de psilídeos após 124 dias, de 37,24 insetos por armadilha (Figura 1).

Efeito da temperatura na flutuação populacional de $\boldsymbol{G}$. brimblecombei e de $\boldsymbol{P}$. bliteus

Observando-se os adultos de $\boldsymbol{G}$. brimblecombei e associando-os com as variáveis meteorológicas do início das avaliações até o final do mês de março, com 62 dias de avaliação (31/03/05), em que as temperaturas mínimas e máximas médias permaneceram em torno de 19 e $31^{\circ} \mathrm{C}$, respectivamente, as populações permaneceram baixas (três psilídeos/ armadilha) e, a partir de 75 dias de avaliação (13/04/05), notou-se que ambas as populações aumentaram, atingindo aos 151 dias de avaliação (28/06/05) 132 psilídeos/armadilha, conforme as temperaturas mínimas

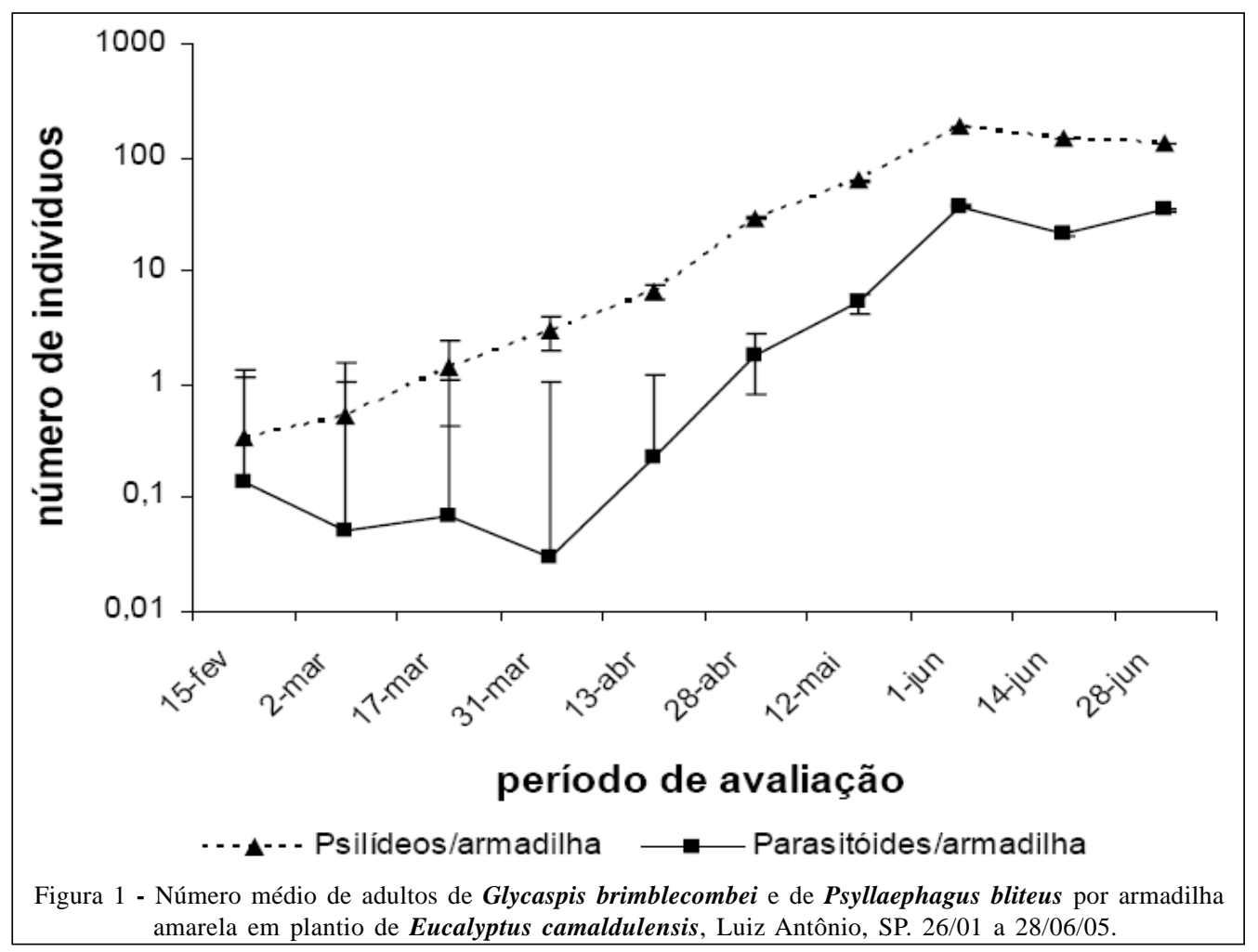

Ciência Rural, v.38, n.8, nov, 2008. 
e máximas médias diminuiram $\left(10\right.$ e $\left.27^{\circ} \mathrm{C}\right)$. O mesmo ocorreu para $\boldsymbol{P}$. bliteus, apenas com número médio de insetos menor ( 0,03 parasitóides/armadilha), até aos 62 dias de avaliação e a partir de 75 dias de avaliação atingem 34,51 parasitóides/armadilha, demonstrando que as duas espécies foram diretamente afetadas pela temperatura (Figuras 1 e 2).

No México, a população do psilídeo-deconcha aumentou juntamente com a temperatura no verão, diminuindo constantemente com a chegada do inverno rigoroso e das geadas, segundo RAMIREZ (2003). No entanto, em Guadalajara, Jalisco, onde o inverno não possui geada, foi possível constatar todas as fases do ciclo de vida e os insetos estavam ativos todo o tempo (CIBRIÁN-TOVAR \& IÑIGUEZHERRERA, 2001).

Nos EUA, foram observadas abundantes populações do psilídeo-de-concha relacionadas com altas temperaturas, nos meses mais quentes, correspondentes a maio, junho e julho (DAHLSTEN, 2002). Na Cidade do México, CIBRIÁN-TOVAR et al., (sd), determinaram a flutuação populacional do psilídeo-de-concha utilizando armadilhas amarelas circulares, verificando altas populações em outubro e baixas já no início de novembro, resultantes da brusca diminuição de temperatura e da detecção de parasitóides na área de estudo. PAINE et al. (2000), na Califórnia, monitoraram populações de $\boldsymbol{G}$. brimblecombei em $\boldsymbol{E}$. camaldulensis e observaram as maiores populações no verão, entre os meses de junho, julho e agosto, onde as temperaturas foram mais altas.

Neste estudo, observou-se que, quando as temperaturas médias mínimas e máximas diminuíram, ocorreu um aumento nas populações de ambas as espécies, devido às características climáticas no Brasil serem diferentes dos países localizados no Hemisfério Norte, que possuem o inverno com temperaturas severamente baixas e verão com temperaturas amenas. Isso pode proporcionar melhores condições para o desenvolvimento dos insetos no verão, uma vez que no inverno seu desenvolvimento se torna limitado. Já no Brasil, as temperaturas aumentam com a chegada do verão, entretanto, no inverno, com a chegada dos meses mais secos e das temperaturas mais amenas (10 a $27^{\circ} \mathrm{C}$ ), a população do psilídeo-de-concha aumenta. Em condições de laboratório, FIRMINO (2004) verificou que a temperatura de $26^{\circ} \mathrm{C}$ foi mais adequada para o desenvolvimento e a reprodução de $\boldsymbol{G}$ brimblecombei.

No Sudoeste da Austrália, região com altas temperaturas, a espécie Cardiaspina albitextura, que

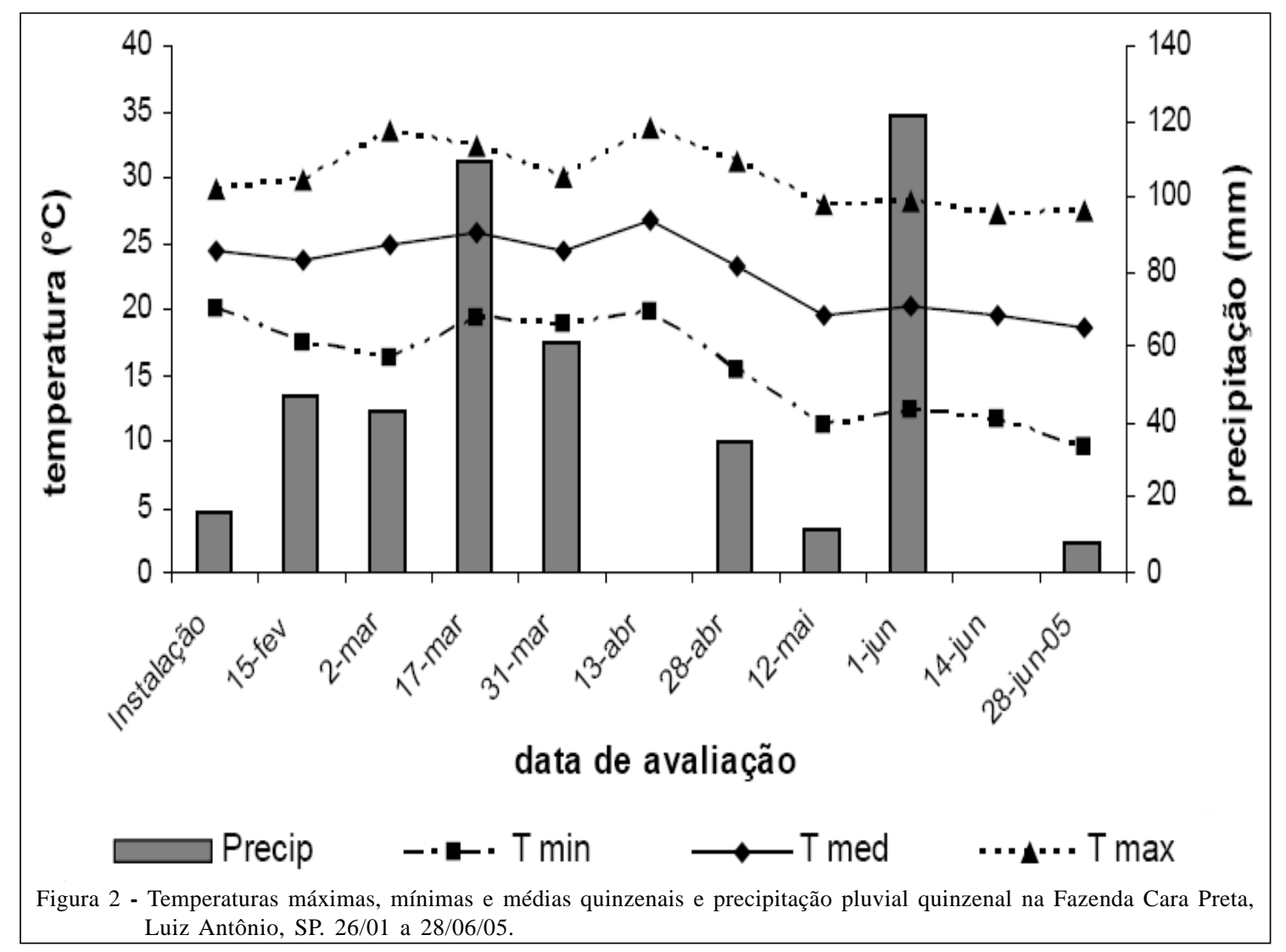

Ciência Rural, v.38, n.8, nov, 2008. 
é principal praga de Eucalyptus blakelyi, possui três gerações por ano e no Sul, região de baixas temperaturas, a espécie leva aproximadamente 18 meses para completar seu ciclo. No entanto, existe a possibilidade de outros fatores particulares de cada região não considerados nos estudos de flutuação populacional estarem afetando a densidade das populações (CLARK, 1962). COULSON \& WRITTER (1990) assinalam que existem fatores que afetam a densidade de uma população, tais como: características meteorológicas, suscetibilidade do hospedeiro, habitat apropriado, parasitismo e enfermidades. BADII et al. (2000) relatam que a predação é um fator importante na regulação da densidade de insetos.

Em relação ao exposto acima e devido à importância da avaliação populacional dos inimigos naturais, CIBRIÁN-TOVAR et al., (sd) utilizaram a mesma metodologia por armadilhas amarelas circulares a fim de determinar a flutuação populacional do parasitóide $\boldsymbol{P}$. bliteus no México e puderam constatar que em outubro a população alcançou nível máximo, diminuindo em novembro, juntamente com a população de $\boldsymbol{G}$ brimblecombei, provavelmente devido às baixas temperaturas registradas. RAMIREZ (2003), estudando diversos aspectos da biologia de $\boldsymbol{P}$. bliteus, observou que seu desenvolvimento é influenciado pela temperatura, sendo mais curto em abril (16,3 dias) com temperatura média de $20,9^{\circ} \mathrm{C}$ e mais longo em novembro (41,6 dias), $\operatorname{com} 16,1^{\circ} \mathrm{C}$.

Efeito da precipitação na flutuação populacional de $\boldsymbol{G}$. brimblecombei e de $\boldsymbol{P}$. bliteus

No início do experimento, quando as chuvas foram mais distribuídas, ambas as espécies se encontraram em baixo número. Porém, a partir de 75 dias de avaliação (13/04/05), quando as chuvas passaram a ser mal distribuídas e desuniformes, proporcionaram melhores condições para que as populações das duas espécies aumentassem (Figuras 1 e 2).
No México, em Cuautitlán Izcalli, a população do psilídeo-de-concha manteve-se alta nos períodos secos e reduziu significativamente nos meses chuvosos, demonstrando que houve uma relação entre a precipitação pluviométrica e a infestação de $\boldsymbol{G}$. brimblecombei, (RAMIREZ et al., 2002). Já RAMIREZ (2003), no Vale do México, pôde concluir, por meio de estudos realizados para flutuação populacional de $\boldsymbol{G}$. brimblecombei, que a precipitação pluvial não teve efeito significativo na variação das populações, apenas foram observadas diminuições na quantidade de insetos, principalmente nos meses de junho e julho.

Nas Ilhas Maurício, África, verificou-se uma relação entre a redução das populações de $\boldsymbol{G}$. brimblecombei com a chegada de períodos de chuvas intensas.Além disso, estudos realizados na Austrália com Glycaspis baileyi indicaram que o clima é um importante fator de regulação da população destes insetos e de outras espécies de psilídeos (SOOKAR et al., 2003).

Nesse caso, devido ao fato de não existir períodos distintos de seca e chuva no decorrer do experimento, não foi possível determinar qualquer interferência significativa entre a flutuação das duas espécies com a precipitação (Tabela 1).

Além disso, seria necessário repetir essas avaliações em outras regiões ou por períodos mais longos para se comprovar esses resultados.

\section{CONCLUSÕES}

Este estudo verificou que as populações de G. brimblecombei e de $\boldsymbol{P}$. bliteus possuem correlação inversamente proporcional em função da temperatura. Portanto, as infestações aumentam conforme ocorre a diminuição da temperatura, com picos nos meses de inverno. Não houve correlação entre ambas as espécies com a precipitação pluviométrica no período avaliado.

Tabela 1 - Valores de correlação linear de Pearson e significância para o período total de avaliação entre o número médio de adultos de Glycaspis brimblecombei, e de Psyllaephagus bliteus com temperaturas máxima, mínima e média e precipitação pluvial em plantio de Eucalyptus camaldulensis, Luiz Antônio SP, 26/01 a 28/06/2005.

\begin{tabular}{cccc}
\hline No médio de insetos & T máx & T min & T méd \\
\hline adultos Gb & & & $-0,83^{*}$ \\
r & $-0,76^{*}$ & $-0,81^{*}$ & 0,0029 \\
P & 0,0109 & 0,0042 & 0,9480 \\
adultos Pb & & & $-0,79^{*}$ \\
r & $-0,70^{*}$ & $-0,79 *$ & 0,0065 \\
P & 0,0237 & 0,0069 & $0,06^{\text {ns }}$ \\
\hline
\end{tabular}

Ciência Rural, v.38, n.8, nov, 2008. 


\section{AGRADECIMENTOS}

Os autores expressam seus agradecimentos à empresa florestal Votorantim Celulose e Papel-VCP, Unidade Luíz Antônio, pelo apoio na instalação e na condução do experimento, com especial referência aos funcionários: biólogo Sérgio A. da Silva e ao técnico Florestal Donizete A. de Oliveira.

\section{REFERÊNCIAS}

BERTI FILHO, E. et al. Ocorrência de Psyllaephagus bliteus Riek (Hymenoptera: Encytidae) no Brasil. Revista de Agricultura, Piracicaba, v.78, n.3, p.304, 2003.

BADII, M.H. et al. Fundamentos y perspectivas de control biológico. In: - Dinámica poblacional. San Nicolás de los Garza, Nuevo León, México: UANL, 2000. p.167174 .

CIBRIAN-TOVAR, D.; HERRERA, G.I. Manual para la identificación y manejo de las pragas y enfermedades forestales del estado de Jalisco. Documento técnico PRODEFO, Guadalajara, Jalisco, n.32, p.23-29, 2001.

CIBRIAN-TOVAR, D. et al. Introducción y antecedentes sobre Glycaspis brimblecombei y de su parasitoide Psyllaephagus bliteus. Manual Técnico Operativo-Tema I. Chapingo: Universidad Autónoma Chapingo, División de Ciencias Forestales, s.d. 85p.

CLARK, L.R. The general biology of Cardiaspina albitextura (Psyllidae) and its abundante in relation to weather and parasitism. Australian Journal of Zoology, v.10, n.4, p.537586, 1962.

COULSON, R.N.; WRITTER, J.A. Entomología forestal. Ecología y control. México: Editorial Limusa D.F., 1990. 751p.

DAHLSTEN, D.L. et al. Parasitoid wasp controls glue gum psyllid. California Agricultura, Oakland, v.52, n.1, p.3134, 1998.

DAHLSTEN, D.L. et al. Pest notes: Eucalyptus redgum lerp psyllid. Oakland: University of California Agricultural Natural Resources Publications, 2003. N.7460, p.1-4.
DAHLSTEN, J.D. Center for biological control. Berkeley: University of California, 2002. 34p. (IPM Education and publications).

DREISTADT, S.H.; GILL, R.J. Pest notes: Eucalyptus redgum lerp psyllid. Oakland: University of California Agricultural Natural Resources Publications, 1999. N.7423, p.1-8.

ELLIOTT, H.J. et al. Insect pests of Australian forests. Melborne: Inkata, 1998. 214p.

FIRMINO, D.C. Biologia do psilídeo-de-concha Glycaspis brimblecombei Moore (Hemiptera: Psyllidae) em diferentes espécies de eucalipto e em diferentes temperaturas. 2004. 49f. Dissertação (Mestrado em Proteção de Plantas) - Faculdade de Ciências Agronômicas, Universidade Estadual Paulista, Botucatu.

GALLO, D. et al. Manual de entomologia agrícola. Piracicaba: Fealq, 2002. 920p.

GILL, R.J. New state records: Redgum lerp psyllid, Glycaspis brimblecombei. California Pest and Disease, n.17, p.7-8, 1998.

PAINE, T.D. et al. UC scientists apply IPM techniques to new eucalyptus pests. California Agriculture, Oakland, v.54, n.6, p.8-13, 2000.

RAMIREZ, A.L.G. et al. Análisis del efecto de las condiciones ambientales en la fluctuación poblacional del psílido del eucalipto en el estado de México. Cuautitlán Izcalli: Editorial Habana, 2002. 5p.

RAMIREZ, A.L.G. Fluctuacion poblacional del psilido del eucalipto Glycaspis brimblecombei y el efecto del control biológico con la avispa parasitóide Psyllaephagus bliteus. 2003. 45f. Tesis de Maestria (Entomología Agrícola) - Ingeniera Agrícola-Facultad de Estudos Superiores Cuautitlan, Edo. de México.

SOOKAR, P. et al. The redgum lerp psyllidae, Glycaspis brimblecombei, a new pest of Eucalyptus sp. in Mauritius. In: ANNUAL MEETING OF AGRICULTURAL SCCIENTISTS, 7., 2003, Réduit, Mauritius. Proceedings... Réduit: AMAS, 2003. p.327-332. Capturado em 18 out. 2004. Online. Disponível em: www.gov.mu/portal/sites/ncb/moa/farc/ amas2003/pdf/p3.pdf 